\title{
The Obstinate Refractory and Resistance Hypertension
}

\author{
Raj Kamal Choudhry ${ }^{*}$, Amrendra Kumar Singh
}

JLN Medical College, Aryabhatta University, Bhagalpur, Bihar, India

Email address:

rajkamalbgp@yahoo.com (R. K. Choudhry)

${ }^{*}$ Corresponding author

\section{To cite this article:}

Raj Kamal Choudhry, Amrendra Kumar Singh. The Obstinate Refractory and Resistance Hypertension. American Journal of Internal Medicine. Vol. 8, No. 5, 2020, pp. 211-214. doi: 10.11648/j.ajim.20200805.13

Received: April 17, 2020; Accepted: August 3, 2020; Published: August 20, 2020

\begin{abstract}
Resistant hypertension (RHTN) is relatively common with an estimated prevalence of $10-20 \%$ of treated hypertensive patients. It is defined as blood pressure (BP) $>140 / 90 \mathrm{mmHg}$ treated with $\geq 3$ antihypertensive medications, including a diuretic, if tolerated. Refractory hypertension is a novel phenotype of severe antihypertensive treatment failure. The proposed definition for refractory hypertension, i.e. BP $>140 / 90 \mathrm{mmHg}$ with use of $\geq 5$ different antihypertensive medications, including a diuretic and a mineralocorticoid receptor antagonist (MRA) has been applied inconsistently. In comparison to RHTN, refractory hypertension seems to be less prevalent than RHTN. This review focuses on current knowledge about this novel phenotype compared with RHTN including definition, prevalence, mechanisms, characteristics and comorbidities, including cardiovascular risk. In patients with RHTN excess fluid retention is thought to be a common mechanism for the development of RHTN. Recently, evidence has emerged suggesting that refractory hypertension may be more of neurogenic etiology due to increased sympathetic activity as opposed to excess fluid retention. Treatment recommendations for RHTN are generally based on use and intensification of diuretic therapy, especially with the combination of a long-acting thiazide-like diuretic and an MRA. Based on findings from available studies, such an approach does not seem to be a successful strategy to control BP in patients with refractory hypertension and effective sympathetic inhibition in such patients, either with medications and/or device based approaches may be needed.
\end{abstract}

Keywords: Aldosterone, Antihypertensive Treatment Failure, Treatment Resistance, Sympathetic Activity, Volume Dependent

\section{Introduction}

With the introduction of antihypertensive pharmacological treatment in the last century, evidence emerged that the blood pressure (BP) response to treatment varies between individuals and that some patients require more medications to control their BP than others. The terms "refractory" and "resistant" hypertension were first used more than six decades ago to characterize such patients [1].

Initially, both terms were interchangeably applied to identify patients with difficult-to-treat hypertension [2, 3]. However, in 2008 the American Heart Association issued a scientific statement to provide a consensus for the definition for resistant hypertension (RHTN). Recently, the term refractory hypertension has been proposed to refer specifically to patients failing maximal antihypertensive treatment defined as lack of BP control despite treatment with at least 5 different antihypertensive agents, including a diuretic and a mineralocorticoid receptor antagonist (MRA) [4].

\section{Discussion}

\section{Resistant hypertension}

As defined in a scientific statement by the American Heart Association Professional Education Committee of the Council for High Blood Pressure Research on Resistant Hypertension, RHTN is defined as uncontrolled BP $(>140 / 90$ $\mathrm{mmHg}$ ) despite use of $\geq 3$ antihypertensive medications, including a diuretic, if tolerated $[5,6]$.

Refractory Hypertension

Refractory hypertension has been suggested to refer specifically to patients who are failing maximal antihypertensive treatment. This recently proposed definition is based on failure to control $\mathrm{BP}>140 / 90 \mathrm{mmHg}$ despite use 
of $\geq 5$ different antihypertensive medications, including a long-acting thiazide-like diuretic, and a mineralocorticoid receptor antagonist [7].

Prevalence

Resistant hypertension

The prevalence of RHTN from various cohorts, including outcome-based trials, registry data, and population studies is estimated to be approximately $10-20 \%$ of patients being treated for hypertension.

Refractory Hypertension

The prevalence of refractory hypertension has been defined in a limited number of studies. The prevalence of refractory hypertension as $2.7 \%$ of patients referred to a hypertension specialty clinic for uncontrolled RHTN, based on having never achieved blood pressure control with use of $\geq 5$ or more medications, including chlorthalidone $25 \mathrm{mg}$ daily and spironolactone $25 \mathrm{mg}$ daily after 3 follow up visits confirmed by 24-hr ambulatory BP monitoring [9-11].

Refractory hypertension was defined as lack of BP control while taking $\geq 5$ antihypertensive medications. However, only $18 \%$ of patients considered as being refractory were being treated with an MRA.

In conclusion, more studies using a standardized definition of refractory hypertension, that is patients who are failing multiple-drug regimens that include both a diuretic and a mineralocorticoid receptor antagonist, are needed.

Pseudo-resistant and pseudo-refractory hypertension

Resistant hypertension

The term apparent RHTN is used when common causes of pseudo-resistant hypertension, such as inaccurate BP measurements, medication non-adherence, under treatment, and white coat effect have not been excluded. Accounting for these causes of pseudo-resistant or pseudo-refractory hypertension will provide more accurate estimates of prevalence rates of true RHTN and true refractory hypertension. It is estimated that combined causes of pseudoresistant hypertension likely account for approximately half of cases of apparent RHTN [12-14].

Table 1. Causes of pseudo resistant hypertension

\begin{tabular}{ll}
\hline Causes of pseudoresistant hypertension \\
\hline 1$)$ & White coat effect \\
$2)$ & Inaccurate Blood Pressure measurement \\
$3)$ & Medication non-adherence \\
$4)$ & Under treatment \\
\hline
\end{tabular}

Causes of pseudo-resistant hypertension:

White coat effect

White coat effect is common in patients with RHTN and is defined as uncontrolled BP in clinic but controlled at home by 24-hr ambulatory BP monitoring.

Role of blood pressure measuring technique

Poor BP measuring technique is a common underlying cause for falsely diagnosed RHTN and refractory hypertension.

Medication non-adherence

Medication non-adherence may be the most common cause of apparent RHTN. In an evaluation of patients with
RHTN referred for renal denervation (RDN).

Refractory hypertension

To what extent the white coat effect, poor BP measurement technique, and medication non-adherence contribute to apparent refractory hypertension is only partially known. Likely the occurrence of these causes of pseudo-refractory hypertension will be at least as common as in patients with RHTN. Under treatment is, by definition, excluded based on an obligatory use of $\geq 5$ different antihypertensive medications including a long-acting thiazide-like diuretic and an MRA.

Mechanisms

Mechanisms of Resistant hypertension

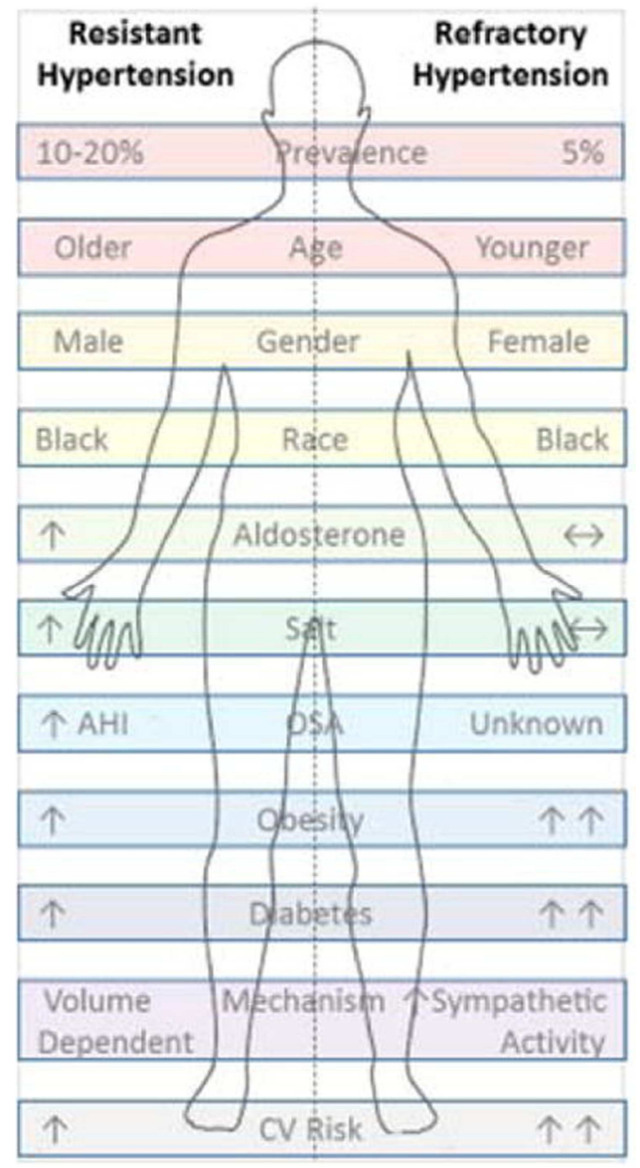

Figure 1. Resistant versus refractory.

RHTN is thought to be attributable in large part to excess fluid retention. This pathophysiologic link is suggested by the pivotal role that intensification of diuretic therapy plays in achieving BP control in patients on multiple antihypertensive medications. Studies have shown that intensification of diuretic therapy, guided by impedance cardiography to measure thoracic fluid content or by indirect biochemical indices of intravascular volume such as natriuretic peptide levels, significantly improves BP control. The etiology of this excess fluid retention is no doubt multifactorial, including older age, obesity, chronic kidney disease (CKD), black race, hyperaldosteronism, and excessive dietary sodium intake [15]. 
Factors associated with refractory hypertension compared with controlled resistant hypertension.

Mechanisms of Refractory Hypertension

Reasons for failure of antihypertensive therapy in patients with refractory hypertension remain poorly understood, but heightened sympathetic activity is widely viewed as a major contributor to treatment failure. Clinical studies suggest that refractory hypertension is less likely to be volume-dependent and more likely to be neurogenic in etiology. Patients with refractory hypertension seem not to have excess fluid retention compared to patients with RHTN [16-19]. These findings are supported by results from impedance cardiography showing that thoracic fluid content is reduced in patients with refractory hypertension. One prospective study by Dudenbostel et al. showed that overactivation of the renin-angiotensin-aldosterone system and rates of hyperaldosteronism are less likely to play a role in patients with refractory hypertension compared to patients with resistant hypertension in general. In that study, Furthermore, in contrast to patients with RHTN, individuals with refractory hypertension, by definition, are failing intensive therapy, including use of combined a long-acting thiazidelike diuretic and an MRA. Combined, these findings argue against volume overload as a major cause of refractory hypertension.

Characteristics and Comorbidities

Resistant hypertension

Compared to patients with more easily controlled hypertension, patients with RHTN are more likely older, Black, and obese. Comorbidities commonly associated with RHTN include chronic kidney disease, type 2 diabetes, hyperaldosteronism, obstructive sleep apnea, and cardiovascular (CV) and cerebrovascular disease.

Refractory Hypertension

Compared to patients requiring multiple medications, but whose BP is controlled, i.e., controlled RHTN, patients with refractory tend to be more often of black race, female, and younger.

Treatment

Resistant hypertension

Treatment of RHTN is largely predicated on intensification of diuretic therapy after failing to control BP with use of a standardized three-drug regimen of an angiotensin converting enzyme inhibitor or angiotensin receptor blocker, a calcium channel blocker, and thiazide-like diuretic. Toward that end, combined use of a long-acting thiazide-like diuretic and an MRA can provide substantial add-on antihypertensive benefit.

This recommendation is based on studies demonstrating the effectiveness of the MRA spironolactone as a fourth antihypertensive agent for treatment of RHTN and on studies indicating superiority of chlorthalidone over hydrochlorothiazide (HCTZ) in terms of antihypertensive benefit. However, with treatment of chlorthalidone and spironolactone, the underlying fluid retention could be overcome with substantial improvement in BP.

Over the last decade, numerous studies have demonstrated the efficacy of the MRA spironolactone for treatment of RHTN.

Refractory Hypertension

How to better treat refractory hypertension has not yet been determined. These patients are failing all commonly used classes of antihypertensive agents, including intensive diuretic therapy with a long-acting thiazide diuretic and an MRA. Preliminary findings suggest that true antihypertensive failure may be partially neurogenic in etiology [20].

\section{Conclusion}

Refractory hypertension is a novel phenotype of antihypertensive treatment failure defined by uncontrolled BP on five or more antihypertensive medications, including a longacting thiazide diuretic and an MRA. This phenotype seems to be rare, comprising only about $5 \%$ of patients referred to a hypertension specialty clinic for uncontrolled RHTN. Like RHTN in general, refractory hypertension is more common in Blacks, patients with obesity, and type 2 diabetes.

However, compared to RHTN, patients with refractory hypertension tend to be younger and more often female. Although longitudinal assessments are so far lacking, crosssectional studies indicate that patients with refractory hypertension are at high risk of congestive heart failure, stroke, CV disease and chronic kidney disease [21, 22]. Underlying mechanisms of true antihypertensive treatment failure may differ from the causes of RHTN in that refractory hypertension may be neurogenic in etiology rather than being volume-overloaded, as is characteristic of RHTN. This observation is supported by the benefit of intensified diuretic therapy, including the use of a MRA for treatment of RHTN in contrast to refractory hypertension, where patients are failing all classes of treatment, including MRAs [23-26].

If the underlying cause of refractory hypertension is attributable to heightened sympathetic output, development of more effective sympatholytic therapies, including devicebased approaches such as renal nerve denervation or baroreceptor stimulation, may provide an effective treatment avenue for this high-risk patient group.

\section{References}

[1] Van Dyne JR. Iproniazid in the treatment of resistant hypertension. A preliminary report on twenty intractable cases. J Am Geriatr Soc. 1960; 8: 454-62. [PubMed] [Google Scholar].

[2] Lee RE, et al. Therapeutically refractory hypertension: causative factors, and medical management with chlorothiazide and other agents. Ann Intern Med. 1958; 49 (5): 1129-37. [PubMed] [Google Scholar].

[3] Calhoun DA, et al. Resistant hypertension: diagnosis, evaluation, and treatment. A scientific statement from the American Heart Association Professional Education Committee of the Council for High Blood Pressure Research. Hypertension. 2008; 51 (6): 1403-19. [PubMed] [Google Scholar]. 
[4] Acelajado MC, et al. Refractory hypertension: definition, prevalence, and patient characteristics. J Clin Hypertens (Greenwich) 2012; 14 (1): 7-12. [PMC free article] [PubMed] [Google Scholar].

[5] Calhoun DA, et al. Refractory hypertension: determination of prevalence, risk factors, and comorbidities in a large, population-based cohort. Hypertension. 2014; 63 (3): 451-8. [PMC free article] [PubMed] [Google Scholar].

[6] Dudenbostel T, et al. Refractory Hypertension: Evidence of Heightened Sympathetic Activity as a Cause of Antihypertensive Treatment Failure. Hypertension. 2015; 66 (1): 126-33. [PMC free article] [PubMed] [Google Scholar].

[7] de la Sierra A, et al. Clinical features of 8295 patients with resistant hypertension classified on the basis of ambulatory blood pressure monitoring. Hypertension. 2011; 57 (5): 898902. [PubMed] [Google Scholar].

[8] Sim JJ, et al. Characteristics of resistant hypertension in a large, ethnically diverse hypertension population of an integrated health system. Mayo Clin Proc. 2013; 88 (10): 1099-107. [PMC free article] [PubMed] [Google Scholar].

[9] Persell SD. Prevalence of resistant hypertension in the United States, 2003-2008. Hypertension. 2011; 57 (6): 1076-80. [PubMed] [Google Scholar].

[10] Egan BM, et al. Uncontrolled and apparent treatment resistant hypertension in the United States, 1988 to 2008. Circulation. 2011; 124 (9): 1046-58. [PMC free article] [PubMed] [Google Scholar].

[11] Modolo R, et al. Refractory and resistant hypertension: characteristics and differences observed in a specialized clinic. J Am Soc Hypertens. 2015; 9 (5): 397-402. [PubMed] [Google Scholar].

[12] Grigoryan L, Pavlik VN, Hyman DJ. Characteristics, drug combinations and dosages of primary care patients with uncontrolled ambulatory blood pressure and high medication adherence. J Am Soc Hypertens. 2013; 7 (6): 471-6. [PMC free article] [PubMed] [Google Scholar].

[13] Muxfeldt ES, et al. True resistant hypertension: is it possible to be recognized in the office? Am J Hypertens. 2005; 18 (12 Pt 1): 1534-40. [PubMed] [Google Scholar].

[14] Pickering TG, et al. Recommendations for blood pressure measurement in humans and experimental animals: part 1: blood pressure measurement in humans: a statement for professionals from the Subcommittee of Professional and Public Education of the American Heart Association Council on High Blood Pressure Research. Circulation. 2005; 111 (5): 697-716. [PubMed] [Google Scholar].

[15] Bhatt H, et al. Prevalence of pseudoresistant hypertension due to inaccurate blood pressure measurement. J Am Soc Hypertens. 2016; 10 (6): 493-9. [PMC free article] [PubMed] [Google Scholar].

[16] Jung O, et al. Resistant hypertension? Assessment of adherence by toxicological urine analysis. J Hypertens. 2013; 31 (4): 766-74. [PubMed] [Google Scholar].

[17] Strauch B, et al. Precise assessment of noncompliance with the antihypertensive therapy in patients with resistant hypertension using toxicological serum analysis. J Hypertens. 2013; 31 (12): 2455-61. [PubMed] [Google Scholar].

[18] Weitzman D, et al. Prevalence and factors associated with resistant hypertension in a large health maintenance organization in Israel. Hypertension. 2014; 64 (3): 501-7. [PubMed] [Google Scholar].

[19] Hwang AY, Dave C, Smith SM. Trends in Antihypertensive Medication Use Among US Patients With Resistant Hypertension, 2008 to 2014. Hypertension. 2016; 68 (6): 1349-1354. [PubMed] [Google Scholar].

[20] Fadl Elmula FE, et al. Adjusted drug treatment is superior to renal sympathetic denervation in patients with true treatmentresistant hypertension. Hypertension. 2014; 63 (5): 991-9. [PubMed] [Google Scholar].

[21] Williams B, et al. Spironolactone versus placebo, bisoprolol, and doxazosin to determine the optimal treatment for drugresistant hypertension (PATHWAY-2): a randomised, doubleblind, crossover trial. Lancet. 2015; 386 (10008): 2059-68. [PMC free article] [PubMed] [Google Scholar].

[22] Siddiqui M, et al. White Coat Effect Is Uncommon in Patients With Refractory Hypertension. Hypertension. 2017 [PMC free article] [PubMed] [Google Scholar].

[23] Khosla N, et al. Are chlorthalidone and hydrochlorothiazide equivalent blood-pressure-lowering medications? J Clin Hypertens (Greenwich) 2005; 7 (6): 354-6. [PubMed] [Google Scholar].

[24] Gaddam K, et al. Rapid reversal of left ventricular hypertrophy and intracardiac volume overload in patients with resistant hypertension and hyperaldosteronism: a prospective clinical study. Hypertension. 2010; 55 (5): 1137-42. [PMC free article] [PubMed] [Google Scholar].

[25] Gaddam KK, et al. Characterization of resistant hypertension: association between resistant hypertension, aldosterone, and persistent intravascular volume expansion. Arch Intern Med. 2008; 168 (11): 1159-64. [PMC free article] [PubMed] [Google Scholar].

[26] Taler SJ, Textor SC, Augustine JE. Resistant hypertension: comparing hemodynamic management to specialist care. Hypertension. 2002; 39 (5): 982-8. [PubMed] [Google Scholar]. 